\title{
Evidence for feature perturbations
}

\author{
GEORGE WOLFORD and KIT HING SHUM \\ Dartmouth College, Hanover, New Hampshire 03755
}

\begin{abstract}
Feature perturbations were proposed by Wolford (1975) to explain the pattern of errors generated by subjects in experiments involving speeded presentations. These experiments were carried out to test the notion of feature perturbations in a fairly direct fashion. The logic of the experiments involved the creation of an artificial alphabet in which feature perturbations, if they occurred, would lead to predictable error patterns. The results of the three experiments supported the notion of feature perturbations. Evidence was also found for errors which resulted from items that were correctly identified but that were reported in an incorrect order. These latter errors were shown to be distinct from errors resulting from feature perturbations.
\end{abstract}

Over the years, there have been many experiments on visual information processing, most of them using some form of speeded presentation. One consistent feature of a wide variety of such experiments is that subjects make substantial numbers of errors. The high error rates were of some surprise to early investıgators, many of whom thought they were examining aspects of the reading process. During reading, skilled readers presumably make relatively few errors. It seems plausible that if we can determine why subjects make errors in our experiments, we will have a better understanding of how visual information is processed.

A number of fundamental constructs have been postulated to account for the occurrence of errors. One is the failure to extract all of the information from the display that is necessary for identification. (This construct is explicit or implicit in many theories of visual information processing; see Shiffrin \& Geisler, 1973, for example.) In Shiffrin and Geisler's model, the necessary information consists of features. Failure to extract all of the features of a letter often leads to an error. The logic of the first construct, however, does not require features as the necessary form of information. Spatial frequencies, whole letters, etc., would work as well (i.e., some portion of the relevant spatial frequency information might not be extracted). A second, often proposed construct is the decay of necessary information from a transient storage system such as an icon (e.g., Estes \& Taylor, 1964). A third construct postulates a variety of capacity limitations such as a limited capacity shortterm memory considered to be a necessary intervening step between extraction and report (e.g., Sperling,

We wish to thank Carol Anne Fowler for her valuable advice and comments at all phases of the reported research. The research was supported, in part, by NIMH Grant MH33179-01. Reprint requests should be addressed to George Wolford, Department of Psychology, Dartmouth College, Hanover, New Hampshire 03755.
1959). Other models generally represent elaborations or mixtures of these constructs.

Wolford (1975) proposed a perturbation model as an alternative way of explaining the patterns of errors observed in a variety of speeded paradigms. The perturbation model was motivated by the failure of existing constructs to provide a natural account of some of the more powerful variables, particularly those relating to lateral interference. The model postulates that a major cause of errors in paradigms using speeded presentations is that the features in a display tend to move or drift, resulting in a loss of relative feature ordering as the display is being processed. The model provided a good, quantitative account of the data from a variety of paradigms. However, there is as yet a lack of direct verification of the perturbation process, despite the fact that several investigators have used the notion of perturbations in their models of information processing (see Krueger, 1978, and Krumhansl \& Thomas, 1977). The experiments that are reported below were designed to test the notion of feature perturbations fairly directly. Before describing the experiments, we will consider the effects of some of the variables that affect visual processing and were instrumental in formulating the perturbation model. The basic assumptions of the perturbation model will be reviewed and a series of experiments designed to test these assumptions will be described. The final discussion section considers the implications of the obtained findings for other models of visual processing.

\section{Retinal Locus}

There has been a renewed interest in the study of retinal locus as an important variable in letter identification. Several investigators recently have designed experiments in which the influence of retinal locus can effectively be partialed out from other variables. Based on the findings of these experiments, a coherent 
picture of the effect of retinal locus is emerging. Two main points characterize the picture. The first is that accuracy decreases and latency increases as the stimulus is moved from the center of the fovea to the periphery (Banks, Bachrach, \& Larson, 1977; Eriksen \& Schultz, 1977; Shaw, 1977; Wolford \& Hollingsworth, 1974a). The second main point is that the retinal gradient (i.e., the performance gradient associated with a letter's retinal location) is far steeper if the stimulus in question is surrounded by other items than if it appears singly (Mackworth, 1965; Wolford, 1975, Figure 2). For example, in the latter study the retinal gradient for letters presented in isolation is fairly flat, decreasing from $94 \%$ at the center of the fovea to $68 \%$ at $2.34 \mathrm{deg}$ from the center in either direction. For a letter flanked by an unreported item on either side, performance dropped from $84 \%$ to $24 \%$ over the same distance. The gradient for letters surrounded by other letters approaches the threshold functions presented in Alpern (1962).

\section{Lateral Interference}

As described above, accuracy in identifying a letter is markedly reduced if the letter is flanked by noise elements or other letters. This effect, known as lateral interference, has several well-documented properties: (1) One interesting property is that the interference is strongly asymmetric. The theoretical nature of the asymmetry is in dispute (see Banks, Bachrach, \& Larson, 1977), but empirically the effect is quite clear. A targeted letter is less likely to be identified if another letter or noise item is placed on the peripheral side of the target letter than if the same noise item is placed on the foveal side of the target letter (Banks et al., 1977; Bouma, 1973; Shaw, 1969; Wolford \& Hollingsworth, 1974b; among others). It should be noted that a noise item on the foveal side of a letter does provide some interference and that placing noise items on both sides of the target causes more of a performance decrement than a single item on either side (Wolford, 1975). (2) The greater the separation between target and noise, the less interference there is (Collins \& Eriksen, 1967). According to Collins and Eriksen, items separated by a degree or more of visual angle exert little or no influence on one another. Eriksen and Schultz (1977) and others have found evidence for interference at longer separations, but there is no question that interference does decrease markedly over the first degree. Effects at larger separations are probably due primarily to response competition. (3) Lateral interference is more pronounced in the periphery than in the fovea (Estes \& Wolford, 1971; Kolers \& Rosner, 1960; Matthews, 1973). This point may be equivalent to the second point raised about retinal locus in the preceding section. (4) Lateral interference can be obtained by presenting every other letter dichoptically, indicating that at least part of the interference is produced more centrally than the retina (Taylor \& Brown, 1972). Finally, (5) some letters are more susceptible to lateral interference effects than others. Letters with their discriminative features on the right side ( $F, R, P$, etc.) are more susceptible to interference on the right side than are symmetric letters ( $\mathrm{A}, \mathrm{H}$, etc.), especially when presented in the right visual field (Estes, Allmeyer, \& Reder, 1976; Wolford \& Hollingsworth, 1974b).

\section{Processing/Report Order}

Even when retinal locus and lateral interference are controlled, an item's position in the instructed processing/report order exerts a strong influence on performance. Items further out on the order are reported less accurately than items at the beginning. This effect tends to be quite powerful (Estes, Allmeyer, \& Reder, 1976; Wolford \& Hollingsworth, 1974a). In the two cited studies, the processing and report orders were probably highly correlated. The concept of report order has considerable surface validity. It is not clear, however, that there exists a factor, processing order, which is independent of report order. It is possible to obtain a processing order effect in a detection task with retinal locus controlled (Wolford \& Hollingsworth, 1974a-Experiments 2 and 3), but the effect is not as pronounced as in a report paradigm. It seems that processing and report are related factors, with the instruction to report amplifying the effect of processing order. Although the concept of a processing order seems to imply a serial component in visual processing, it need not do so, as there exist decisional explanations of such effects (Gardner, 1973).

\section{Nature of Stimuli}

It has been amply demonstrated over the past several years that confusability plays a major role in performance. The more confusable the target item is with the noise items in a detection task, the less accurate subjects will be in identifying the target (Estes, 1972; Gardner, 1973; Krumhansl \& Thomas, 1977). Wolford and Hollingsworth (1974c) analyzed the errors from a whole-report paradigm and found that a significant percentage of the errors represent visual confusions.

\section{PERTURBATION MODEL}

The perturbation model (Wolford, 1975) was formulated to provide a quantitative account of the effects of the variables described in the preceding section and to provide the account in as simple a fashion as possible. The majority of existing models of letter identification deliberately avoided dealing with retinal locus, lateral interference, and report paradigms in order to concentrate on other aspects of the data, typically the effects of confusability. One exception to this is the interactive channels model (Estes, 1972), which does deal specifically with retinal locus and 
lateral interference. The interactive channels model and other models and constructs will be discussed in more detail following a description of the perturbation model and the experiments.

The key concept of the perturbation model is that the features that make up the various items in the display tend to migrate over time (i.e., information regarding the relative location of various features is distorted). If certain properties are assumed regarding the nature of the perturbation process, then many of the effects of the above variables can be accounted for on the basis of feature perturbation. There are three primary assumptions regarding the perturbation process: the amount of perturbation varies as a function of retinal locus, perturbations in the direction of the fovea are more likely than perturbations toward the periphery, and the probability of a feature migrating to some location is inversely related to its distance from the original location (i.e., features are more likely to move short distances than long distances).

An intuitive feel for the model may be enhanced by considering its account of lateral interference. Two items that are sufficiently close to one another will end up with some intermixture of features, leading to a deterioration in performance. Since several features of the actual stimulus will also be present, errors will be similar to that stimulus leading to confusion errors. Wolford (1975) contains a formal description of the model.

The perturbation model was able to provide a good quantitative account of the data from a variety of paradigms with relatively few parameters. A weakness of previous tests of the model is that the general idea of feature perturbation was chosen post hoc to account for data that had already been collected. This is not to imply that the good fits were trivial; the general framework of the model was formulated with awareness of the nature of the effects that had to be accounted for. We are convinced that the way to further test the model is not to generate new predictions from it or to extend it "as is" to other paradigms. First, the model is quite powerful and will necessarily work well in most simple cases; sec- . ondly, any failure would be difficult to isolate within the model. The failure could arise from the basic notion of feature perturbation, but it could just as easily arise from a poor choice of decision rule, an inadequate feature set, or a poor choice of theoretical functions in implementing the model. What is needed is some relatively direct evidence that the basic assumption of feature perturbation is sensible. It is toward that end that the experiments described below were carried out.

\section{EXPERIMENT 1}

The purpose of the first experiment was to create a situation in which feature perturbations would be

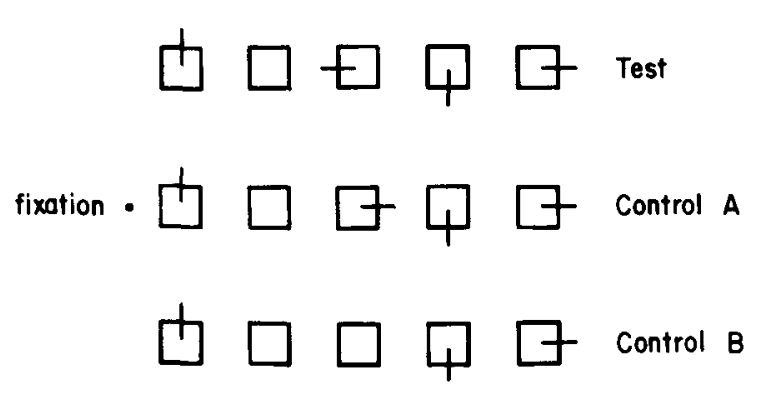

Figure 1. Configuration of stimuli used in Experiment 1.

noticeable if they occurred. The essence of the design is illustrated in Figure 1. On each trial, the subjects were presented with a set of five characters to the right of a fixation point. The character set consisted of five different members: a square with no ticks (a "blank") and squares with ticks bisecting one of the four sides referred to as "up," "right," "down," and "left." Looking at the three display types in Figure 1, our primary interest concerned performance on the second position, which was always a "blank." The display types were distinguished by the type of character in the following position. Test displays always contained a "left" to the right of the "blank," control- $A$ displays always contained a "right," and control Bs, another "blank." There was an additional set of displays with the critical "blank" occurring in Position 3, and there also were a number of random displays to make any pattern in the displays less noticeable.

The primary prediction is that the "blank" in Position 2 of the test displays will be reported as a "right" more often than chance. The "right" response results from a feature migration of the tick in Position 3 to Position 2. According to the perturbation model, this migration is relatively likely, since the tick is close to the square in Position 2 and the move would be in the direction of the fovea. A feature migration to Position 2 in the control-A displays would be less likely due to the increased distance of the tick from Position 2. A feature migration of a tick from Position 3 to 2 is not possible for the control-B displays, as there is no tick in Position 3.

There is another possible source of error in this experiment that will be referred to as whole-symbol movement. There is considerable evidence from report tasks and from short-term memory experiments that subjects misreport the positions of stimuli. Estes, Allmeyer, and Reder (1976) found that these wholesymbol shifts were also likely to occur in the direction of the fovea. For our present study, this would mean that if the subjects made an error in Position 2, the incorrect response would be the stimulus that had occurred at Position 3 more often than chance.

Putting feature migrations together with wholesymbol movement in our experiment, we would predict that on test displays subjects would make both 
"right" (feature perturbation) and "left" (wholesymbol movement) errors with greater than chance frequency. On control-A trials, "right" (wholesymbol movement) errors would occur with greater than chance frequency. On control-B trials, neither feature migration nor whole-symbol movement could be detected as errors, and the errors that are made should be randomly distributed among the four alternatives. As a corollary to the preceding predictions, we would expect an ordering of the three display types with respect to overall probability correct. Control-B trials should be the best, since neither feature migration nor whole-symbol movement is a problem; control-A trials should be next best, since only whole-symbol movement is a factor; and test trials should be the worst, since both types of error are expected to occur.

\section{Method}

Subjects and Apparatus. Twenty-five undergraduates were recruited from an introductory psychology course at Dartmouth College. They received course credit for participating in the experiment.

The stimulus materials were presented in a Scientific Prototype three-channel tachistoscope (Model GA) that was modified with a rapid card changer on one of the channels. The stimuli were presented along the horizontal median of a lighted rectangular field which subtended a visual angle of $7.82 \mathrm{deg}$ in width and $1.68 \mathrm{deg}$ in height. One field (the fixation field) contained a circular fixation point measuring $.073 \mathrm{deg}$ in diameter and centered with respect to the rectangular field. The luminance of all of the fields was adjusted to $5 \mathrm{fL}$. The fixation field was visible at all times except when the stimuli were presented. The total illumination of the laboratory was provided by a $7-\mathrm{W}$ bulb shielded from the subject.

Stimulus materials. There were a total of 40 stimulus cards, each one containing a set of five possible characters: a "blank," an "up," a "right," a "down," and a "left." The 40 stimuli were divided into 4 groups of 10 each. In the first group, the variation condition, the five possible characters appeared in an essentially random sequence. In the second group, the test condition, a target square appeared either at the second position or the third. This target was always a "blank," and it was always followed by a "left." The same was true for the third group, the control-A condition, except that the square immediately after the target blank square was always a "right." In the final group, the control-B condition, the target blank square was always followed by another blank square. The final three stimulus groups were partitioned into two sets, one-half with the critical "blank" at Position 2 and the other half with the critical "blank" at Position 3. The stimuli were printed on white $5 \times 8$ in. note cards. The dimensions of the stimuli are given in Figure 2.



Figure 2. Dimensions of stimuli in degrees of visual angle used in Experiment 1.
Procedure. Each subject received three presentations of the deck of 40 cards. The cards were shuffled before each presentation. A trial began with the fixation field in view and the experimenter saying "Ready." The subject then initiated a trial by depressing a hand-held microswitch. The stimulus field appeared with no delay for $100 \mathrm{msec}$ and was followed by a return to the fixation field. Following each exposure, the subject was to report aloud the identity of each of the five characters in a left-to-right order, guessing if necessary. Subjects were reminded that a blank square could occur and that they could call for a short break whenever they wanted. No feedback was provided, and the subjects were not told that they were going to see the same stimuli three times. The experiment lasted approximately $45 \mathrm{~min}$.

\section{Results and Discussion}

The first set of analyses was limited to the critical target on each of the displays. Recall that the target symbol was always a "blank" and that this target was always flanked on the right by a "left" in the test condition, by a "right" in the control-a condition, and by another "blank" in the control-B condition. In half of these displays, the target appeared in the second position in from the fixation point; in the other half, in the third position. The primary results consist of the raw frequency, totaled across all 25 subjects, of each possible response type for the critical position as a function of display type. Those results are presented in Table 1. For all cases, "blank" would be a correct response.

The pattern of results conformed to our predictions. The fact that "left" errors were at chance for control-A displays provides assurance that the critical error types did not result from some "generalized horizontal error." For statistical analysis purposes, the frequencies have been summed over the cases where the critical "blank" appeared in Positions 2 and 3. Since there were two predicted sources of error for the test displays, it is not completely accurate to compare each one against .25. (For a discussion of this issue, see Wolford \& Hollingsworth, 1974c). Thus, the reliability of the critical error types was computed in two ways: against the average of "up" and "down" errors for the same display types (cf. Wolford \& Hollingsworth, 1974c), and against the respective response categories in control-B displays.

In the first case, the average frequency of "up" and "down" errors was calculated for the individual subjects. This would be the baseline against which the "left" and "right" errors were compared using a paired-t statistic. For both the test and the control-A conditions, the frequency of "right" errors was significantly greater than the baseline (for $24 \mathrm{deg}$ of freedom, $t=2.21$ with $p=.035$, and $t=3.93$ with $\mathrm{p}=.0007$, respectively), whereas for the control-B condition it was not $(t=-.90$ with $p=.62)$. On the other hand, the frequency of "left" errors was significantly greater than the baseline only for the test condition (the values of $t$ for the three conditions being $5.18,-.36$, and -.88 , respectively). Next, using the corresponding error frequencies in the control-B 
Table 1

Distribution of Responses for Target Positions in Experiment 1

\begin{tabular}{|c|c|c|c|c|c|c|c|c|c|c|}
\hline \multirow[b]{3}{*}{ Condition } & \multicolumn{10}{|c|}{ Response } \\
\hline & \multicolumn{5}{|c|}{ Position 2} & \multicolumn{5}{|c|}{ Position 3} \\
\hline & Blank & Up & Right & Down & Left & Blank & Up & Right & Down & Left \\
\hline $\begin{array}{l}\text { Test } \\
\text { Control A } \\
\text { Control B }\end{array}$ & $\begin{array}{l}202 \\
238 \\
274\end{array}$ & $\begin{array}{l}20 \\
25 \\
30 \\
\end{array}$ & $\begin{array}{l}57^{*} \\
62^{*} \\
22 \\
\end{array}$ & $\begin{array}{l}20 \\
25 \\
28 \\
\end{array}$ & $\begin{array}{l}76^{*} \\
25 \\
21 \\
\end{array}$ & $\begin{array}{l}144 \\
179 \\
240\end{array}$ & $\begin{array}{l}39 \\
41 \\
38\end{array}$ & $\begin{array}{l}55^{*} \\
72^{*} \\
31\end{array}$ & $\begin{array}{l}48 \\
44 \\
35 \\
\end{array}$ & $\begin{array}{l}89 * \\
39 \\
31 \\
\end{array}$ \\
\hline
\end{tabular}

*Significantly different from baseline at .05 level.

condition as the baseline, paired-t comparisons indicated significantly more "left" as well as "right" errors in the test condition (for $24 \mathrm{deg}$ of freedom, $t=5.48$ for "left" and $t=4.70$ for "right" errors). As for the control-A condition, only "right" errors were significantly more frequent than in the control-B condition ( $t=.74$ for "left" and $t=4.79$ for "right" errors). Although there were several $t$ tests and not all of the tests were independent of one another, there did not appear to be any better way of directly testing our predicted outcomes.

Thus, both analyses confirmed that in the test condition, both "left" and "right" errors were overrepresented, while in the control-A condition, only "right" errors were more frequent. This was exactly what was predicted: In the test condition, a feature migration would result in "right" errors and a wholesymbol transposition would result in "left" errors, while in the control-A condition, both sources of errors resulted in "rights." As was noted earlier, the fact that "left" errors were not predominant in the control-A condition suggested that the present pattern of results was not due to some indiscriminate "horizontal" errors but, rather, only to those specifically postulated. The above analyses were performed on the combined data from Positions 2 and 3. Looking at those positions separately, the same pattern was obtained at each position, but the feature perturbation errors were somewhat less prominent at Position 3 than at Position 2.

Another prediction of the perturbation model concerns feature movement within characters. Since all of the displays were presented to the right of the fixation point, we would expect feature movement to occur primarily from the right to the left. This would imply that "right" symbols should on occasion be identified as "left" due to feature migration. "Lefts," on the other hand, should rarely become "rights," since the feature movement occurs in the wrong direction. To examine this prediction, we looked at "rights" and "lefts" which occurred anywhere in any of the display types, including the random variations. There was a total of 773 errors made to "right" symbols in the experiment. Of those errors, "left" was, as predicted, by far the most common response, occurring $34 \%$ of the time, with "blank," "up," and "down" responses occurring 14\%, 27\%, and $25 \%$ of the time, respectively. There was a total of 621 errors occurring to "left" symbols. "Right" responses were not the most frequent and occurred $24 \%$ of the time, with "blank," "up," and "down" responses occurring $21 \%, 26 \%$, and $29 \%$ of the time, respectively. To test whether this pattern of results was statistically significant, a paired $t$ was used to compare the conditional probabilities of a "left" error given a "right" symbol, and of a "right" error given a "left" symbol. Since these were percentages, an arcsine transformation (Winer, 1971) was applied to the data before computing t. For $24 \mathrm{deg}$ of freedom, the value of $t$ was 2.83 , which is significant at the .01 level. The predictions of the perturbation model concerning feature migration were, therefore, again supported.

More information concerning whole-symbol movement can be obtained by examining positions in the displays other than just the critical positions used in Table 1. For Positions 2, 3, and 4 in all displays, the probability was computed that an error made at the nth position was the symbol that occurred at position $n-1$ or $n+1$. These data are displayed in Figure 3. As can be seen clearly, whole-symbol move-



Figure 3. Percentages of whole-symbol movements in the Peripheral (shaded) vs. Foveal (unshaded) direction as a function of position in report order for Experiment 1. 
ment is far more likely to occur in the direction of the fovea. This asymmetry also was found in Estes, Allmeyer, and Reder (1976). The statistical reliability of this result was tested by performing a paired- $t$ comparing the conditional probabilities of foveal vs. peripheral whole-symbol transposition after an arcsine transformation. The result was again significant $(t=8.09$ with $p$ approaching zero).

There were several auxiliary predictions that could be generated based on the notion of feature migration. Subjects might be expected to see squares with multiple ticks, ticks in between squares, and ticks in the middle of squares. No subject reported any of these, but the subjects had been given a thorough description of the character set and the labels to use in reporting the characters, which probably precluded the above options. Another prediction might be labeled an autocorrelation of errors. On the test displays, if the subject makes the "right" error on the critical positions and if the error is the result of a feature migration from the adjacent square as claimed, then the adjacent square should be labeled a "blank," since it has shed its tick. Looking at the appropriate trials, "blank" responses did not occur significantly more often than chance. There are several problems with this particular analysis, however. The response "blank" was underrepresented in the data as a whole, perhaps indicating some bias on the part of the subjects. In addition, the errors on those cases of interest could have been influenced by whole-symbol movement from the periphery.

The final comment on this experiment concerns the use of squares with ticks as the character set. While the notion that letter identification is based on the extraction and analysis of "features" has gained considerable popularity in models of letter recognition, the identity of the features that are critical in recognizing letters has remained somewhat in doubt. No existing choice of a feature set does an outstanding job of accounting for performance, although several are adequate. Our choice of squares with ticks was motivated by two major considerations: (1) If there are features, vertical and horizontal line segments are likely members of the set, and (2) if the ticks move as predicted, relational information is preserved. For instance, if the tick from a "left" does migrate to a "blank," there are still four right angles. The situation would be somewhat different if something like triangles with horizontal ticks had been used instead. Then if a tick migrated from a "left" to a "blank," the spatial ordering of acute and obtuse angles would be destroyed. Since the identity of the features involved in letter identification is uncertain, a set was chosen which would maximize the probability of observing migration.

According to the perturbation model, there is no reason to expect that only the ticks and not the sides of the squares will migrate. However, since the sub- jects know that five squares are presented on each trial and confine their report to that restriction, the migration of other parts of the squares should not affect any of the preceding analyses.

\section{EXPERIMENT 2}

The purpose of the second study was to attempt to distinguish between feature perturbation and wholesymbol movement. The distinction between feature perturbation and whole-symbol movement errors is of considerable importance in trying to model visual information processing. If both types of errors are the result of the same underlying process, then the notion of feature perturbation should be replaced, since it greatly underpredicts whole-symbol errors. Some other process would then need to be formulated which could account for both types of errors. On the other hand, if the two types of errors are separable, perhaps occurring at different stages of processing, then it would make sense to save the basic perturbation model to account for feature errors and to postulate a separate mechanism for dealing with wholesymbol movement.

It seems quite plausible that feature perturbations occur (or only have an effect) prior to object identification and that whole-symbol movements occur at some later stage, subsequent to object identification. There is considerable evidence, for instance, of lettertransposition errors in short-term memory experiments.

Consistent with the possibility of the two error types occurring at different stages, we would expect them to be responsive to different variables. In particular, feature perturbation errors should be affected by sensory variables such as visual field, distance from the center of the fovea, spacing between characters, etc., while whole-symbol movement errors should be sensitive to more cognitive variables such as instructed report order, memory load, and subsequent interference.

In particular, there was a hint in the first experiment that one source of whole-symbol movement errors was what might be referred to as a "sink" effect (Estes, Allmeyer, \& Reder, 1976). If subjects fail to identify the character in, say, Position 2 on some trial, but do identify the characters in Positions 3-5, there may be some tendency to report the identified characters in Positions 2-4, guessing at Position 5, even though the subjects are instructed to report the items in the correct order. The sink effect in the above example would lead to three wholesymbol movement errors, and all three errors would be toward the beginning of the report order.

Report order and retinal locus were also nearer to the center of the fovea. It was impossible to decide, therefore, whether the whole-symbol errors in the first study were influenced by the report order or by retinal locus. 
In Experiment 2, we used a 2 by 2 design with visual field varied orthogonally with report order. A similar design was used by Estes and Wolford (1971). Visual field was varied randomly throughout a session, with half of the displays being presented entirely in the right visual field (RVF) (as in Experiment 1) and half in the left visual field (LVF). One group of subjects was instructed to report the displays from the fixation point toward the periphery (in-out) and a second group was instructed to report from the periphery toward the fixation point (out-in). Our hypothesis was that feature perturbation errors would occur in the direction of the fovea in both visual fields and independently of instructed report order. Whole-symbol movement errors were predicted to occur in the direction of the beginning of the instructed report order. For the in-out order, they would occur in the direction of the fovea, but for the out-in group, they would occur in the opposite direction.

\section{Method}

Subjects and Apparatus. Twenty subjects were recruited from among Dartmouth students enrolled in an introductory psychology course. They were assigned randomly to two groups of 10 each, one group for each report order. The apparatus and display the same as in Experiment 1.

Stimulus materials. The character set and size of characters were the same as in Experiment 1. We decided to focus on intracharacter feature perturbations (i.e., "lefts" being reported as "rights" and vice versa) rather than intercharacter perturbations, as in the first experiment. As described in a subsequent section, the intracharacter perturbations appear to be more discriminating theoretically, and every trial represents a potential test, so there is less waste.

Since we were concentrating on intracharacter perturbations, the displays were not divided into types as in Experiment 1. Instead, 10 displays were constructed with the five characters chosen at random, subject to the constraint that each display had to contain at least one "left" or "right." A second set of 10 displays was constructed by replacing all "lefts" in the first set with "rights" and all "rights" with "lefts." This switch insures that the "lefts" and "rights" are equal in position, frequency, and surround across this set of 20 displays. These 20 stimulus strings were then positioned on $8 \times 5$ cards so that they would appear in the RVF. A second set of 20 stimulus displays was constructed by positioning the 20 stimulus strings in the LVF with a reverse ordering of the characters (i.e., the matched pair for the two visual fields would have the same characters, beginning with the ones closest to the fixation point and extending outwards). There was a total, then, of $\mathbf{4 0}$ cards, and each subject was shown this set twice.

Procedure. One group was instructed to report in-out and the other out-in. Each subject received 10 practice trials followed by two presentations of the 40 stimulus cards. These were shuffled before being presented for the second time. Other details of presentation, subjects' responding, and data recording were the same as in Experiment 1.

\section{Results and Discussion}

The overall performance levels are displayed in Figure 4. As can be seen, the instructed report order had a marked effect on the serial position curves, with items early in the report order receiving a performance edge. There was also a tendency for the out-in group to

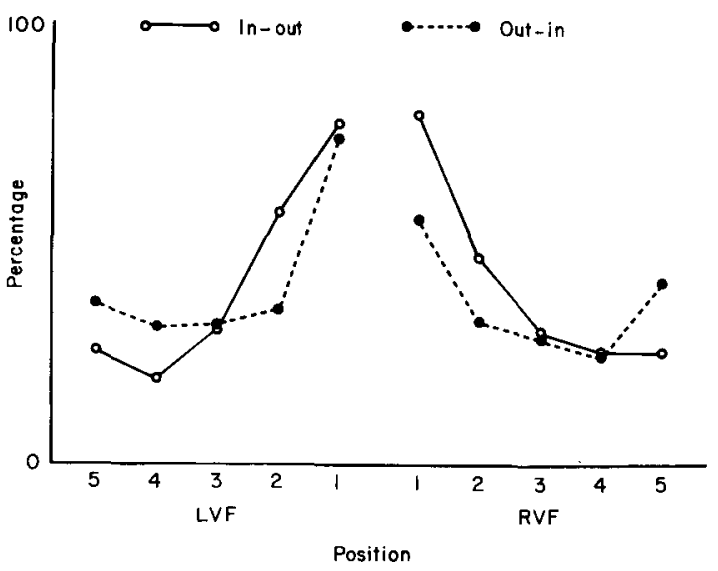

Figure 4. Serial position curves for percentages of correct identification as a function of both left (LVF) vs. right (RVF) visual field and In-Out vs. Out-In report order for Experiment 2.

be lower overall in performance, particularly in the RVF. In fact, two of the subjects in the out-in group were responding at nearly a chance level in the RVF.

The next analysis focused on feature migration errors. The difference between the probability of a "right" error given a "left" in the stimulus ensemble and the probability of a "left" error given a "right" was chosen as an index of feature migration. We predict that features would tend to migrate toward the fovea. This index, therefore, should be negative for the right visual field displays, and positive for the left visual field displays. This was indeed borne out in the results: the mean of the index was $-6.3 \%$ for the right visual field and $6.9 \%$ for the left visual field. (The mean percentages of the two types of errors used in computing the indices are shown in Table 2.) Since the index was based on percentages, the arcsine transformation was applied to these percentages before subjecting the index to statistical analysis. A two-way analysis of variance was performed on the indices, with visual field as a within-subjects factor and report order as a between-subjects factor. The visual field factor was significant $[F(1,18)=4.34$, $\mathrm{p}=.049]$. The report order factor was not significant $[\mathrm{F}(1,18)=2.70, \mathrm{p}=.10]$. There was no interaction between the two factors $[F(1,18)=2.37, p=.10]$.

The final analysis concerned the whole-symbol movement errors. When there is an error, two kinds of whole-symbol movement errors may occur. The subject may report in its place either the adjacent symbol on the side of the fovea or that on the peripheral side. The index chosen was the difference between the probability of such an error in the direction of the fovea and that in the direction away from the fovea. If, as predicted, the direction of wholesymbol movement should vary with report order, then the index would be positive for the in-out report order and negative for the out-in. This prediction was confirmed: For the in-out condition, the mean 
Table 2

Means (in Percentages) for the Feature Migration Index for Experiment 2

\begin{tabular}{|c|c|c|c|c|c|c|c|}
\hline \multirow[b]{3}{*}{ Report Order } & \multicolumn{6}{|c|}{ Retinal Location } & \multirow{3}{*}{$\begin{array}{l}\text { Subtotals for } \\
\text { Report } \\
\text { Order }\end{array}$} \\
\hline & \multicolumn{3}{|c|}{ LVF } & \multicolumn{3}{|c|}{ RVF } & \\
\hline & $P(R / L)$ & $P(L / R)$ & $\begin{array}{c}\mathrm{P}(\mathrm{R} / \mathrm{L}) \\
-\mathrm{P}(\mathrm{L} / \mathrm{R})\end{array}$ & $P(R / L)$ & $P(L / R)$ & $\begin{array}{c}P(R / L) \\
-P(L / R)\end{array}$ & \\
\hline $\begin{array}{l}\text { In-Out } \\
\text { Out-In }\end{array}$ & $\begin{array}{l}39.0 \\
33.3\end{array}$ & $\begin{array}{l}30.4 \\
28.1\end{array}$ & $\begin{array}{l}8.6 \\
5.2\end{array}$ & $\begin{array}{l}21.5 \\
29.7\end{array}$ & $\begin{array}{l}35.3 \\
28.6\end{array}$ & $\begin{array}{r}-13.8 \\
1.1\end{array}$ & $\begin{array}{r}-2.6 \\
3.1\end{array}$ \\
\hline Subtotals for Retinal Location & & & 6.9 & & & -6.3 & \\
\hline
\end{tabular}

Note-P(R/L) stands for the probability of a "right" given a "left" stimulus, conditionalized upon making an error.

Table 3

Means (in Percentages) for the Whole-Symbol Movement Index for Experiment 2

\begin{tabular}{|c|c|c|c|c|c|c|c|}
\hline \multirow[b]{3}{*}{ Report Order } & \multicolumn{6}{|c|}{ Retinal Location } & \multirow{3}{*}{$\begin{array}{c}\text { Subtotals for } \\
\text { Report } \\
1 \quad \text { Order }\end{array}$} \\
\hline & \multicolumn{3}{|c|}{ LVF } & \multicolumn{3}{|c|}{ RVF } & \\
\hline & Foveal & Peripheral & $\begin{array}{c}\text { Foveal } \\
- \text { Peripheral }\end{array}$ & Foveal & Peripheral & $\begin{array}{c}\text { Foveal } \\
- \text { Peripheral }\end{array}$ & \\
\hline $\begin{array}{l}\text { In-Out } \\
\text { Out-In }\end{array}$ & $\begin{array}{l}31.6 \\
25.3\end{array}$ & $\begin{array}{l}21.6 \\
27.9\end{array}$ & $\begin{array}{l}10.0 \\
-2.6\end{array}$ & $\begin{array}{l}35.0 \\
25.2\end{array}$ & $\begin{array}{l}16.4 \\
27.0\end{array}$ & $\begin{array}{r}18.6 \\
-1.8\end{array}$ & $\begin{array}{r}14.3 \\
-2.2\end{array}$ \\
\hline Subtotals for Retinal Location & & & 3.7 & & & 8.4 & \\
\hline
\end{tabular}

Note-Foveal stands for the probability of making a whole-symbol movement in the foveal direction, conditionalized upon making an error.

was 14.3; for the out-in condition, it was -2.2 . Table 3 shows the mean percentages of whole-symbol movement errors in both directions. After an arcsine transformation, the data were analyzed with a twoway analysis of variance similar to the preceding analysis. This time, the prediction was that the wholesymbol movement, occurring at a late stage of processing, would depend on report order. The main effect of report order was significant $[F(1,18)=25.40$, $\mathrm{p}=.0001]$. The main effect of visual field was not significant $[\mathrm{F}(1,18)=2.21, \mathrm{p}=.15]$, and there was no interaction $[\mathrm{F}(1,18)=1.46, \mathrm{p}=.24]$.

The results of Experiment 2 support the contention that feature migrations are distinct from wholesymbol errors. Feature migrations were sensitive to visual field, occurring primarily in the direction of the fovea, and were not sensitive to report order. Whole-symbol movement was not sensitive to visual field but was affected by report order, with the movement occurring toward the beginning of the instructed report order. The pattern of results is also consistent with the notion that feature migrations occur at a sensory level while whole-symbol errors occur at a more cognitive level.

We were concerned about the occasional reversals in the data for the out-in group and the low overall performance level for that group. The subjects in that group reported difficulty in following the report-order instructions. Thus, it is quite possible that many of the errors in the out-in group result from failures to report in the instructed direction. Reporting in the incorrect direction could lead to nonrandom error patterns, particularly at Position 3. This tendency for some subjects to report in the incorrect direction would in fact explain some of the observed reversals.

\section{EXPERIMENT 3}

Experiment 3 was designed to clear up some of the difficulties just mentioned concerning Experiment 2 . We attempted to obtain better adherence to the instructed report order by providing subjects with more trials. This was accomplished by having each subject participate in two sessions rather than one and by using more trials per session. Also, a larger pool of stimulus strings was constructed and the subjects were provided with feedback concerning their performance every 20 trials. The feedback was used to encourage adherence to the instructions.

\section{Method}

Subjects and Apparatus. Twenty new subjects were chosen from the same population used in the previous experiments. Ten subjects were randomly assigned to each report order condition. The apparatus and display conditions were identical to those used in Experiments 1 and 2.

Stimulus materials. There were 120 stimulus cards, each one containing a set of five characters. As before, there were five possible characters: a "blank," an "up," a "right," a "down," and a "left."

Thirty cards were created first so that the set of five squares would appear to the right of the fixation point. The characters were chosen at random, but care was taken to ensure that there was an equal number of each of the five possible characters in each serial position. Thirty more cards were constructed by 
reversing the "lefts" and "rights" of the original 30 . Then, an additional 60 cards were constructed so that the set of five characters would appear to the left of the fixation point and each one was a mirror image of the other sixty, making a total of 120 cards constructed.

Procedure. There were two conditions, with half of the subjects in each. All subjects were required to participate in two sessions (on consecutive days). The same 120 cards were presented for all sessions and for all subjects, but the cards were shuffled before each presentation. A trial began with the fixation field in view and the experimenter saying "Ready," The subject then initiated a trial by depressing a hand-held microswitch. The stimulus field appeared with no delay for $50 \mathrm{msec}$ and was followed by a blank field, which lasted $8 \mathrm{sec}$, and then a return to the fixation field. Following each exposure, the subject was to report aloud the identity of each of the five characters. In the in-out condition, subjects were told to start with the character nearest the center (the fixation point), no matter on which side of the visual field the set of squares might appear; subjects in the out-in condition were told to start with the one furthest from the center. Subjects were reminded that a blank square could occur and that they should guess if necessary.

The subject's responses were typed by the experimenter into a computer which also performed the necessary calculations for providing feedback to the subject. There were two kinds of feedback. First, the session was divided into six blocks of 20 cards each. After each block, the subject was informed of the percentages of the characters that were correctly identified overall and for each serial position. This helped the experimenter monitor whether the subjects were reporting in the appropriate order. A second, more immediate feedback was provided by calculating an average number of correct responses for every consecutive group of five trials. That number was compared with a running average of all the trials so far completed. If the average for the current five trials was greater than the running average, the subject would be given three beeps from the computer. The subject was also told that he or she could call for a break whenever he or she wanted. Each session lasted approximately $50 \mathrm{~min}$.

\section{Results}

The serial position curve for each of the four cells in the 2 by 2 design is presented in Figure 5 . There was no difference between the two visual fields. In fact, performance in the two fields was almost perfectly symmetrical for both report orders. However, the overall shape of the curve differed for the two report orders, that is, the subjects in the in-out condition did better on the first two positions but worse on the last position, as compared with the outin subjects. These curves are similar to those found in Estes and Wolford (1971).

Since this experiment was essentially a replication of Experiment 2, the same analyses were used. For



Figure 5. Serial position curves for percentages of correct identification as a function of both left (LVF) vs. right (RVF) visual field and In-Out vs. Out-In report order for Experiment 3.

the feature migration, the visual field factor was significant $[F(1,18)=6.01, p=.024]$. The mean of the index was $-.7 \%$ for the right visual field and $10.9 \%$ for the left visual field (Table 4 shows the mean percentages of the errors used in computing the indices). The report order factor was not significant $[\mathrm{F}(1,18)=.08, \mathrm{p}=.77]$. There was also a significant interaction between the two factors $[F(1,18)=$ $5.16, \mathrm{p}=.04]$. A look at the means indicated that the interaction was due to the fact that the index was not negative on the right visual field for the Out-In subjects. This could have been due to an overall response bias for "right" on the part of the subjects. However, it was indeed smaller relative to the left visual field.

In the analysis concerning whole-symbol errors, the main effect of report order fell short of significance at the .05 level $[\mathrm{F}(1,18)=1.98, \mathrm{p}=.17]$. The main effect of visual field was not significant $[F(1,18)$ $=.08, \mathrm{p}=.77]$; but there was again a marginally significant interaction $[F(1,18)=4.25, p=.06]$. The mean percentages of whole-symbol errors in both directions are shown in Table 5.

It is interesting to note that in Experiment 2, the feature migration was less significant than the whole-

Table 4

Means (in Percentages) for the Feature Migration Index for Experiment 3

\begin{tabular}{|c|c|c|c|c|c|c|c|}
\hline \multirow[b]{3}{*}{ Report Order } & \multicolumn{6}{|c|}{ Retinal Location } & \multirow{3}{*}{$\begin{array}{c}\text { Subtotals for } \\
\text { Report } \\
\text { Order }\end{array}$} \\
\hline & \multicolumn{3}{|c|}{ LVF } & \multicolumn{3}{|c|}{ RVF } & \\
\hline & $P(R / L)$ & $\mathbf{P}(\mathrm{L} / \mathrm{R})$ & $\begin{array}{c}P(R / L) \\
-P(L / R)\end{array}$ & $\mathrm{P}(\mathrm{R} / \mathrm{L})$ & $\mathbf{P}(\mathbf{L} / \mathbf{R})$ & $\begin{array}{c}\mathrm{P}(\mathrm{R} / \mathrm{L}) \\
-\mathrm{P}(\mathrm{L} / \mathrm{R})\end{array}$ & \\
\hline $\begin{array}{l}\text { In-Out } \\
\text { Out-In }\end{array}$ & $\begin{array}{l}37.6 \\
31.1\end{array}$ & $\begin{array}{l}21.9 \\
25.0\end{array}$ & $\begin{array}{r}15.7 \\
6.1\end{array}$ & $\begin{array}{l}23.8 \\
28.8\end{array}$ & $\begin{array}{l}30.1 \\
23.9\end{array}$ & $\begin{array}{r}-6.3 \\
4.9\end{array}$ & $\begin{array}{l}4.7 \\
5.5\end{array}$ \\
\hline Subtotals for Retinal Location & & & 10.9 & & & -.7 & \\
\hline
\end{tabular}

Note-P(R/L) stands for the probability of a "right" given a "left" stimulus, conditionalized upon making an error. 
Table 5

Means (in Percentages) for the Whole-Symbol Movement Index for Experiment 3

\begin{tabular}{|c|c|c|c|c|c|c|c|}
\hline \multirow[b]{3}{*}{ Report Order } & \multicolumn{6}{|c|}{ Retinal Location } & \multirow{3}{*}{$\begin{array}{c}\text { Subtotals for } \\
\text { Report } \\
\text { Order }\end{array}$} \\
\hline & \multicolumn{3}{|c|}{ LVF } & \multicolumn{3}{|c|}{ RVF } & \\
\hline & Foveal & Peripheral & $\begin{array}{c}\text { Foveal } \\
- \text { Peripheral }\end{array}$ & Foveal & Peripheral & $\begin{array}{c}\text { Foveal } \\
- \text { Peripheral } \\
\end{array}$ & \\
\hline In-Out & 30.1 & 27.2 & 2.9 & 29.8 & 30.6 & -.8 & 1.1 \\
\hline Out-In & 23.6 & 28.5 & -4.9 & 24.8 & 27.1 & -2.3 & -3.6 \\
\hline Subtotals for Retinal Location & & & -1.0 & & & -1.5 & \\
\hline
\end{tabular}

Note-Foveal stands for the probability of making a whole-symbol movement in the foveal direction, conditionalized upon making an error.

symbol movement ( $\mathrm{p}=.049$ and .0001 , respectively), while in the present experiment, the reverse was true, that is, the feature migration was more significant than the whole-symbol movements $(p=.024$ and .17 , respectively). In fact, the latter was not statistically significant. The loss of the whole-symbol effect in Experiment 3 after obtaining highly significant effects in Experiments 1 and 2 may suggest that the whole-symbol effect occurs at a cognitive level over which the subject exercised control. The changes in Experiment 3, such as feedback or number of trials, may have caused subjects to change strategies, thereby altering the whole-symbol effect.

\section{GENERAL DISCUSSION}

The data from the three experiments provide evidence that something like feature perturbation occurs at an early stage in processing. No detailed information is available on the functional nature of the perturbation process, but the fact that the prominence of feature perturbations in Experiment 1 was less in Position 3 than in Position 2 is contrary to the functions postulated in Wolford (1975). Precise functional information is required for the quantitative modeling of the process. Acquiring it is the goal of future research, with particular concern about the effect of retinal locus, character separation and character type on the frequency of perturbations.

The prominence of whole-symbol movement suggests the need for an extension or revision of the perturbation model. The model in its current form does predict some whole-symbol movement errors, since, if some of the features of a symbol migrate to another feature group, there is some probability that that feature group will be identified mistakenly as the subsequent character. The frequency of occurrence of such whole-symbol movement errors which occur that way in simulations of the model falls far short of the frequency in the data. In addition, according to the perturbation model, whole-symbol errors occurring in that way should be a function of visual field, not report order.

Given the nature of whole-symbol movement errors and their dependence on the instructed report order, it seems plausible that they result from the loss of order information subsequent to item identification. Such a process could be added as a later stage to the current perturbation model.

\section{Alternative Theoretical Approaches}

A number of very promising models of visual information processing have appeared over the last several years, and these models employ a number of different constructs in accounting for letter identification data. Evaluating these models based on the obtained data, however, is somewhat unfair, as many of these were specially designed to deal with data from experimental situations somewhat removed from our paradigms. For instance, Shiffrin and Geisler (1973) deliberately restrict their model to situations in which all stimuli are equidistant from the center of the fovea and sufficiently far apart to avoid significant lateral interference. Furthermore, they restrict themselves to the detection paradigm, as do many other investigators.

Unlimited parallel channels model. Their model belongs to a class of models that have been labeled unlimited, independent, parallel channels models (Collins \& Eriksen, 1967; Gardner, 1973; Shiffrin \& Geisler, 1973). In this class of models, the primary sensory contributor to errors is the failure to extract all of the features from any given character. Decisions are made on the basis of the available features of each character. In this situation of imperfect featural information and with the decision rules generally used, interitem confusability exerts considerable influence on performance. The number of features required to identify a target depends directly on the number of features that the targets and noise items have in common. If they share no features, then only one target feature is required to assure perfect performance; if they share several features, then missing target features may well lead to a confusion error. As far as we can tell, the perturbation model is indistinguishable from this class of models when retinal locus is held constant and spacing between items is generous. The unlimited parallel channels models, however, would have to be modified to deal with the data from the current experiments. The feature perturbation errors could not be 
accounted for on the basis of failure to identify some of the features, since no subset of the features of a "blank" make it look like a "right," and since in our experiments the "right" error is dependent on the nature of the adjacent item.

It might be possible to add some sort of parsing mechanism to this class of models and then assume that what happened on some of the test trials in Experiment 1 was that the subject failed to detect one of the vertical lines of the critical stimulus and perhaps the space on its periphery. The parser might then place the vertical line and tick from the adjacent stimulus with the critical stimulus leading to a "right" response.

The intracharacter confusion errors documented in all three experiments, but particularly in Experiments 2 and 3 , argue against the parsing solution just described. The parsing mechanism would allow the models to predict an overabundance of "left" errors to "right" stimuli in the RVF and "right" errors to "left" stimuli in the LVF. That same mechanism, however, would also have to predict an overabundance of the opposite error types in the two visual fields. In other words, the parsing mechanism predicts a generalized horizontal error, whereas the obtained error pattern is quite specific.

Decay. Several models have either implicitly or explicitly assumed that errors are made in tachistoscopic experiments because the information is registered in a transient store and that information decays before all of the items are processed (Sperling, 1959; Estes \& Taylor, 1964). With respect to the data presented, the notion of decay appears to be logically equivalent to the notion of not extracting all of the relevant features from the display. For the same reasons listed above, decay, by itself, will not account for the constellation of effects referred to as feature perturbation errors.

Organizational constructs. Several investigators have presented convincing evidence that the organization of the items in a display has a strong influence on performance. Banks and Prinzmetal (1976) have argued that several existing variables, such as homogeneity of noise elements, may owe their effects to organizational principles. Given the displays used in our experiments, we do not see any way of characterizing the organization of the different display types that would lead to an accurate account of the data. We are impressed, however, by the ability of subjects to report only from the stimulus set used and by the fact that the displays generally appear, phenomenologically, to contain five squares. Given the notion of feature perturbations and the postulate that not all of the features are necessarily extracted, one might expect subjects to see a jumble of features. Some of the overall organization of a display is probably being detected and probably at a very early stage. Banks, Bachrach, and Larson (1977) argued that many existing lateral interference effects could be explained on the basis of the retinal placement of target and noise. Such an argument should not apply to the current research, since all of the display types have similar spatial characteristics. One possibility concerning perturbations is that the features of an item vary in the degree to which they are "bound" together. Good figures might be more "bound," leading to fewer perturbations.

Spatial frequency analysis. Several investigators have proposed that the spatial frequency characteristics of a stimulus form the basis of pattern recognition (Broadbent, 1977; Graham, 1976). Several neural structures have been found which are selectively receptive to various spatial frequencies, and several behavioral outcomes lend themselves to a spatial frequency interpretation. Spatial frequency analysis has not often been applied directly to studies of letter identification, but in a recent publication, Coffin (1978) did so and concluded that spatial frequency analysis did not provide an adequate account of the confusion data from a letter identification paradigm. It is unclear how an analysis of spatial frequencies could produce the error pattern observed in the current work. We are attracted to the idea expressed by Broadbent (1977) and Kinchla (1977) that a preliminary and rapid analysis of the low spatial frequencies may be partly responsible for the perception of overall organization of displays.

Interactive channels model. Besides the perturbation model, the model most relevant to our research was formulated by Estes (1972). The main assumptions of the model are as follows: (1) Information is extracted from visual displays in parallel at the feature level, (2) the density of detectors for each feature type decreases with increasing distance from the center of the fovea, and (3) decisions are made on the basis of the features that have been extracted at the various retinal locations.

The interactive channels model is capable of dealing with the effects of lateral interference and will in many cases make predictions similar to those of the perturbation model. We believe that the interactive channels model could be extended in a natural way to account for some of the effects in the data, but that it (like the previous models) would have to predict that if subjects make "left" errors to "right" characters in the right visual field, then they should also make "right" errors to "left" characters in the right visual field.

Other models. There are several hybrid models which also look promising. Bjork and Murray (1977) have proposed a variant of the interactive channels model to account for a variety of data, but we believe, for the same reasons listed previously, that their model would not provide an adequate account of the current data.

Krumhansl and Thomas (1977) have proposed a model that appears to be a cross between the interactive channels model and the perturbation model. 
They also found in their empirical results some support for the concept of feature perturbations. Since their model subsumes the perturbation model, it could handle our results as well as the original model.

It is somewhat unfair to evaluate other visual information processing models on the basis of our data, since the other models were not formulated to deal with that particular situation. There may be ways that we have overlooked that would allow some other construct or model to deal with the same data as well or better than the perturbation model. Nevertheless, the constellation of effects in our research does fit in well with the notion of feature perturbations.

\section{REFERENCES}

Alpern, M. Muscular mechanisms. In H. Davson (Ed.), The eye (Vol. 3). New York: Academic Press, 1962.

Banks, W. P., BaChrach, K., \& Larson, D. W. The asymmetry of interference in visual letter identification. Perception \& Psychophysics, 1977, 22, 232-240.

Banks, W. P., \& Prinzmetal, W. Configurational effects in visual information processing. Perception \& Psychophysics, 1976, 19, 361-367.

Bjork, E. L., \& Murray, J. T. On the nature of input channels in visual processing. Psychological Review, 1977, 84, 472-484.

Bouma, H. Visual interference in the parafoveal recognition of initial and final letters of words. Vision Research, 1973, 13, 767.782 .

Broadbent, D. E. The hidden preattentive process. American Psychologist, 1977, 32, 109-118.

Coffin, S. Spatial frequency analysis of block letters does not predict experimental confusions. Perception \& Psychophysics, 1978, 23, 69-74.

Collins, J. F., \& Eriksen, C. W. The perception of multiple simultaneously presented forms as a function of foveal spacing. Perception \& Psychophysics, 1967, 2, 369-373.

Eriksen, C. W., \& Schultz, D. W. Retinal locus and acuity in visual information processing. Bulletin of the Psychonomic Society, 1977, 9, 81-84.

Estes, W. K. Interactions of signal and background variables in visual processing. Perception \& Psychophysics, 1972, 12, 278 286.

Estes, W. K., Allmeyer, D. H., \& Reder, S. M. Serial position functions for letter identification at brief and extended exposure durations. Perception \& Psychophysics, 1976, 19, 1-15.

Estes, W. K., \& TAylor, H. A. A detection method and probabilistic models for assessing information processing from brief visual displays. Proceedings of the National Academy of Sciences, 1964, 52, 446-454.
Estes, W. K., \& Wolford, G. L. Effects of spaces on report from tachistoscopically presented letter strings. Psychonomic Science, 1971, 25, 77-79.

GardneR, G. T. Evidence for independent parallel channels in tachistoscopic perception. Cognitive Psychology, 1973, 4, 130-155.

Graham, N. Spatial frequency channels in human vision: Detecting edges without edge detectors. In C. S. Harris (Ed.), Visual coding and adaptability. Hillsdale, N.J: Erlbaum, 1976.

Kinchla, R. A. The role of structural redundancy in the perception of visual targets. Perception \& Psychophysics, 1977, 22, 19-30.

Kolers, P. A., \& Rosner, B. S. On visual masking (metacontrast): Dichoptic observation. American Journal of Psychology, $1960,73,2-21$.

KRUEGer, L. E. A theory of perceptual matching. Psychological Review, 1978, 85, 278-304.

Krumhansl, C. L., \& Thomas, E. A. C. Effect of level of confusability on reporting letters from briefly presented visual displays. Perception \& Psychophysics, 1977, 21, 269-279.

Mackworth, N. H. Visual noise causes tunnel vision. Psychonomic Science, 1965, 3, 67-68.

Matthews, M. L. Locus of presentation and the selective masking effect. Canadian Journal of Psychology, 1973, 27, 343-349.

Shaw, M. L. Reaction time in reading tachistoscopic displays for a memory set item. Perception \& Psychophysics, 1977, 21, 15-25.

Shiffrin, R. M., \& Geisler, W. S. Visual recognition in a theory of information processing. In R. L. Solso (Ed.), Contemporary issues in cognitive psychology: The Loyola symposium. Washington, D.C: Winston, 1973.

SPERLING, G. Information in a brief visual presentation. Unpublished doctoral dissertation, Harvard University, 1959.

TAylor, S. G., \& Brown, D. R. Lateral visual masking: Supraretinal effects when viewing linear arrays with unlimited viewing time. Perception \& Psychophysics, 1972, 12, 97-99.

Winer, B. J. Statistical principles in experimental design. New York: McGraw-Hill, 1971.

Wolford, G. Perturbation model for letter identification. Psychological Review, 1975, 82, 184-199.

Wolford, G., \& Hollingsworth, S. Retinal location and string position as important variables in visual information processing. Perception \& Psychophysics, 1974, 16, 437-442. (a)

Wolford, G., \& Hollingsworth, S. Lateral masking in visual information processing. Perception \& Psychophysics, 1974, 16, 315-320. (b)

Wolford, G., \& Hollingsworth, S. Evidence that short-term memory is not the limiting factor in the tachistoscopic full report procedure. Memory \& Cognition, 1974, 2, 796-800. (c)

(Received for publication November 5, 1979; revision accepted February 19, 1980.) 